\title{
Air pollutants and outpatient visits for influenza-like illness in Beijing, China
}

\author{
Xiaoguang Li ${ }^{1}$, Jie Xu ${ }^{1}$, Wei Wang ${ }^{1}$, Jing-Jin Liang ${ }^{1}$, Zhong-Hua Deng ${ }^{1}$, Juan Du ${ }^{2}$, Ming-Zhu Xie ${ }^{2}$, Xin-Rui Wang ${ }^{2}$ \\ , Yaqiong Liu ${ }^{2}$, Fuqiang Cui ${ }^{\text {Corresp., }}$, Qing-Bin Lu ${ }^{\text {Corresp. } 2}$ \\ ${ }^{1}$ Department of Infectious Diseases, Peking University Third Hospital, Beijing, Beijing, China \\ Department of Laboratorial of Science and Technology, School of Public Health, Peking University, Beijing, Beijing, China \\ Corresponding Authors: Fuqiang Cui, Qing-Bin Lu \\ Email address: cuifuq@bjmu.edu.cn, qingbinlu@bjmu.edu.cn
}

\section{Background}

Air pollution leads to many adverse health conditions, mainly manifested by respiratory or cardiac symptoms. Previous studies are limited as to whether air pollutants were associated to influenza-like illness (ILI). This study aimed to explore the association between air pollutants and outpatient visits for ILI, especially during an outbreak of influenza.

\section{Methods}

Daily counts of hospital visits for ILI were obtained from Peking University Third Hospital between January 1, 2015, and March 31, 2018. A generalized additive Poisson model was applied to examine the associations between air pollutants concentrations and daily outpatient visits for ILI when adjusted for the meteorological parameters.

\section{Results}

There were 35862 outpatient visits at the fever clinic for ILI cases. Air quality index (AQI), PM ${ }_{2.5}, \mathrm{PM}_{10}, \mathrm{CO}$ and $\mathrm{O}_{3}$ on lag 0 days, as well as nitrogen dioxide $\left(\mathrm{NO}_{2}\right)$ and sulfur dioxide $\left(\mathrm{SO}_{2}\right)$ on lagl days, were significantly associated with an increased risk of outpatient visits for ILI from January 2015 to November 2017. From December 2017 to March 2018, on lag0 days, air pollutants $P_{2.5}$ [risk ratio $(R R)=0.971,95 \%$ $\mathrm{Cl}: 0.963-0.979], \mathrm{SO}_{2}(\mathrm{RR}=0.892,95 \% \mathrm{Cl}: 0.840-0.948)$ and $\mathrm{CO}(\mathrm{RR}=0.306,95 \% \mathrm{Cl}: 0.153-0.612)$ were significantly associated with a decreased risk of outpatient visits for ILI. Interestingly, on the lag2 days, all the pollutants were significantly associated with a reduced risk of outpatient visits for ILI except for $\mathrm{O}_{3}$. We did not observe the linear correlations between the outpatient visits for ILI and any of air pollutants, which were instead associated via a curvilinear relationship.

\section{Conclusions}

We found that the air pollutants may be associated with an increased risk of outpatient visits for ILI during the non-outbreak period and with a decreased risk during the outbreak period, which may be linked with the use of disposable face masks and the change of outdoor activities. These findings expand the current knowledge of ILI outpatient visits correlated with air pollutants during an influenza pandemic. 
1 Air pollutants and outpatient visits for influenza-like illness in Beijing, China

2 Xiaoguang $\mathrm{Li}^{1}$, Jie $\mathrm{Xu}^{1}$, Wei Wang ${ }^{1}$, Jing-Jin Liang ${ }^{1}$, Zhong-Hua Deng ${ }^{1}$, Juan Du ${ }^{2}$, Ming-Zhu

$3 \mathrm{Xie}^{2}$, Xin-Rui Wang ${ }^{2}$, Ya-Qiong Liu², Fuqiang Cui²*, Qing-Bin $\mathrm{Lu}^{2 *}$

4 1. Department of Infectious Diseases, Peking University Third Hospital, Beijing 100191, P.R.

$5 \quad$ China.

6 2. Department of Laboratorial of Science and Technology, School of Public Health, Peking $7 \quad$ University, Beijing 100191, P.R. China

9 Corresponding authors:

10 Qing-Bin Lu, M.D.,

11 Department of Laboratorial of Science and Technology, School of Public Health, Peking

12 University, No. 38 Xue-Yuan Road, Haidian District, Beijing 100191, P.R. China. Tel (Fax):

13 +86-10-82805327. Email: qingbinlu@bjmu.edu.cn

14 Fuqiang Cui, Professor, $\mathrm{PhD}$,

15 Department of Laboratorial of Science and Technology, School of Public Health, Peking

16 University, No. 38 Xue-Yuan Road, Haidian District, Beijing 100191, P.R. China. Tel (Fax):

17 +86-10-82801518. Email: cuifuq@,bjmu.edu.cn

\section{Running title}

Air pollutants and ILI 


\section{Abstract}

\section{Background}

25 Air pollution leads to many adverse health conditions, mainly manifested by respiratory or cardiac symptoms. Previous studies are limited as to whether air pollutants were associated to influenza-like illness (ILI). This study aimed to explore the association between air pollutants and outpatient visits for ILI, especially during an outbreak of influenza.

\section{Methods}

Daily counts of hospital visits for ILI were obtained from Peking University Third Hospital between January 1, 2015, and March 31, 2018. A generalized additive Poisson model was applied to examine the associations between air pollutants concentrations and daily outpatient visits for ILI when adjusted for the meteorological parameters.

\section{Results}

There were 35862 outpatient visits at the fever clinic for ILI cases. Air quality index (AQI), $\mathrm{PM}_{2.5}, \mathrm{PM}_{10}, \mathrm{CO}$ and $\mathrm{O}_{3}$ on lag0 days, as well as nitrogen dioxide $\left(\mathrm{NO}_{2}\right)$ and sulfur dioxide $\left(\mathrm{SO}_{2}\right)$ on lag1 days, were significantly associated with an increased risk of outpatient visits for ILI from January 2015 to November 2017. From December 2017 to March 2018, on lag0 days, air pollutants $\mathrm{PM}_{2.5}$ [risk ratio $\left.(\mathrm{RR})=0.971,95 \% \mathrm{CI}: 0.963-0.979\right], \mathrm{SO}_{2}(\mathrm{RR}=0.892,95 \% \mathrm{CI}$ : $0.840-0.948)$ and $\mathrm{CO}(\mathrm{RR}=0.306,95 \% \mathrm{CI}: 0.153-0.612)$ were significantly associated with a

41 decreased risk of outpatient visits for ILI. Interestingly, on the lag2 days, all the pollutants were

42 significantly associated with a reduced risk of outpatient visits for ILI except for $\mathrm{O}_{3}$. We did not 43 observe the linear correlations between the outpatient visits for ILI and any of air pollutants, 
44 which were instead associated via a curvilinear relationship.

\section{Conclusions}

46 We found that the air pollutants may be associated with an increased risk of outpatient visits for

47 ILI during the non-outbreak period and with a decreased risk during the outbreak period, which

48 may be linked with the use of disposable face masks and the change of outdoor activities. These

49 findings expand the current knowledge of ILI outpatient visits correlated with air pollutants

50 during an influenza pandemic.

51

52

53

54

55

56

57

58

\section{Summary}

We found that the air pollutants may be associated with the increased risk of outpatient visits for

ILI during the non-outbreak period while it revealed the associations with the decreased risk

during the outbreak period. 


\section{Background}

Outdoor fine particulate matter (particulate matter with an aerodynamic diameter less than $2.5 \mu \mathrm{m}, \mathrm{PM}_{2.5}$ ) exposure causes 4.2 million deaths and a loss of greater than 100 million disability-adjusted life years each year (Schraufnagel et al. 2018). In China, over 1.3 billion people are exposed to high amounts of ambient fine particulate matter and subsequently have high health risks (Song et al. 2017). Concentrations of air pollutants are much higher in winter than those in summer, and the average concentrations in northern China are higher than those in southern China (Chen et al. 2018).

Air pollution leads to many adverse health conditions, mainly manifested by respiratory or cardiac symptoms (Schraufnagel et al. 2018). In China, many studies have already demonstrated that exposure to $\mathrm{PM}_{2.5}, \mathrm{PM}_{10}$ and other air pollutants, such as nitrogen dioxide $\left(\mathrm{NO}_{2}\right)$ and sulfur dioxide $\left(\mathrm{SO}_{2}\right)$, can induce great adverse impacts on human health, such as ischemic heart disease, cerebrovascular disease, chronic obstructive pulmonary disease (COPD), and lung cancer (Chen et al. 2018; Li et al. 2017; Song et al. 2017; Xia et al. 2017; Zhang et al. 2015). One study reported that $\mathrm{PM}_{2.5}$ and $\mathrm{PM}_{10}$ levels were associated with an increased risk of arrhythmia admissions among 26 Chinese cities, especially in elderly and diabetic patients (Zheng et al. 2018).

Airborne pollution particles may provide "condensation nuclei" to which virus droplets attach, suggesting that the air pollutants may be related to the risk of influenza-like illnesses (ILIs) or pneumonia caused by influenza virus. However, few studies have shown how ambient $\mathrm{PM}_{2.5}$ concentrations might increase the risk of exposure and subsequent transmission of 
80

81

82

influenza in China (Chen et al. 2017; Liang et al. 2014; Lu et al. 2020). It has been demonstrated ambient PM2.5 concentrations increased the number of ILIs during the flu season in Beijing, China (Feng et al. 2016). Air pollutants, especially $\mathrm{PM}_{2.5}, \mathrm{PM}_{10}, \mathrm{CO}$ and $\mathrm{SO}_{2}$, can increase the risk of ILI in Jinan, China (Su et al. 2019). In the USA, short-term exposure to elevated $\mathrm{PM}_{2.5}$ concentrations have induced greater healthcare utilization for acute lower respiratory infections in children and adults (Horne et al. 2018; Strosnider et al. 2018), which has also been associated with increased rates of culture-negative pneumonia and influenza (Croft et al. 2018). While in another study in Hefei, China, $\mathrm{PM}_{10}$ was negatively associated with clinical ILI, while $\mathrm{PM}_{2.5}$ were positively associated with clinical ILI (Liu et al. 2019). There were no associations between air pollutants $\left(\mathrm{PM}_{2.5}, \mathrm{PM}_{10}\right.$ and $\left.\mathrm{NO}_{2}\right)$ and ILI risk for people aged over 25 years old in Nanjing (Huang et al. 2016). The conflicting results were observed and these studies are limited as to whether air pollutants contribute to the number of ILI cases and did not adjust for the potential confounding biases by meteorological parameters. In addition, there was no outbreak of influenza virus during the time series described above. It is necessary to further confirm the effects of influenza outbreaks on the associations between air pollutants and the risks of ILI outpatient visits.

The purpose of this study was to explore the associations between air pollutants and outpatient visits for ILI, especially during an outbreak of ILI, which may provide a scientific basis for objectively evaluating air pollution effects and preventing acute respiratory tract infections. 
101

102

103

104

105

106

107

108

109

110

111

112

113

114

115

116

117

118

119

120

121

\section{Methods}

\section{Data collection}

The counts of daily outpatient visits for ILI were obtained from Peking University Third Hospital, Beijing, China. Daily outpatient visits at fever clinics with a primary diagnosis of ILI (ICD-10 J11.101) between January 1, 2015, and March 31, 2018 were extracted from hospital database. Peking University Third Hospital severed more than 4.22 million outpatients and 300,000 emergency patients every year. Fever outpatients have been ranked top two in the general hospitals in Beijing, which can cover patients in urban and suburban areas. The research protocol was approved by the human ethics committee of Peking University Third Hospital (IRB00006761-M2018221). The inform consent was not applicable, as this manuscript only uses quantitative data from hospital system which use unique identifiers and not names nor contact information.

Patients less than 15 years old were excluded from this analysis. The concentrations of air pollutants, including air quality index (AQI), $\mathrm{PM}_{2.5}, \mathrm{PM}_{10}, \mathrm{SO}_{2}, \mathrm{CO}, \mathrm{NO}_{2}$ and $\mathrm{O}_{3}$, were collected between January 1, 2015 and March 31, 2018, from the Data Center in the Ministry of Ecological Environment of the People's Republic of China. Daily mean temperature, mean relative humidity, mean wind speed and sunshine time were provided by the National Meteorological Information Center. Twenty-four-hour means were used for all air pollutants except $\mathrm{O}_{3}$, which is conventionally measured at a maximum interval of eight hours.

The higher the AQI value, the greater the level of air pollution and the greater the health concern. AQI is divided into six categories, including good (0-50), moderate (51-100), unhealthy 
122 for sensitive groups (101-150), unhealthy (151-200), very unhealthy (2201-300) and hazardous $123(>300)$.

124 Statistical analysis

125 Correlations between air pollutant concentrations and meteorological parameters during the

126 study period were estimated using Spearman's correlation coefficients due to the abnormal distributions of all these variables.

The study course was classified into two periods: a non-outbreak period from January 2015 to November 2017 and an outbreak period from December 2017 to March 2018 according the distribution of the number of ILI outpatient visits.

Daily outpatient visits for ILI, air pollutant concentrations and meteorological parameters were merged by date to be analyzed with a time-series design. A generalized additive Poisson model was applied to examine the associations between each air pollutant concentration and the counts of daily outpatient visits for ILI adjusted for daily mean temperature, mean relative humidity, mean speed wind and sunshine time and days of the week. The meteorological parameters were controlled using natural spline smoothing functions. Dummy variables were used for days of the week to control for potential confounding factors. To control for any lag effect, the concentrations of air pollutants on the current day (lag0) and the previous five days (lag1-5) were incorporated into the model.

Associations were expressed as the percent change and 95\% confidence interval (CI) in the count of daily outpatient visits associated with a per- $10 \mathrm{mg} / \mathrm{m}^{3}$ increase in the air pollutant concentrations. All analyses were performed using R Programming Language V.3.2.2 (R 
143 144 College Station, TX, United States).
146

147

148

149

150

151

152

153

Development Core Team) with the "mgcv" and "nlme" packages and Stata 14.0 (Stata Corp LP,

\section{Results}

\section{Outpatient visits}

There were 35862 outpatient visits at the fever clinic for ILI cases from January 2015 to March 2018 in the study (Table 1). Among all the ILI cases, 53.9\% of the patients were 25-60 years old. The median (interquartile range) count of daily ILI-attributed outpatient visits was 17 (12-28). The yearly outpatient visits at the fever clinic are shown in Supplemental Table 1. The number of ILI cases increased rapidly along with the year, especially in the first three months of 2018. From Figure 1, four peaks were observed in January-February 2015, 2016, 2017 and 2018. It is noteworthy that the number of ILI cases at the abnormal peak in 2018 was nearly three times as large as those at the other three peaks.

\section{Description of exposure and meteorological parameters}

The time series of $\mathrm{AQI}$ and six air pollutants, $\mathrm{PM}_{2.5}, \mathrm{PM}_{10}, \mathrm{SO}_{2}, \mathrm{CO}, \mathrm{NO}_{2}$, and $\mathrm{O}_{3}$, are shown in Figure 2. The day-distribution of AQI grades was significantly different across the four years $(\mathrm{P}<0.001)$, as shown in Supplemental Figure 1. The AQI value in 2018 was lower than in other years, showing no peak. The number of days of hazardous pollution were $15,9,7$ and 0 in 2015, 2016, 2017 and 2018, respectively. Air pollutants $\mathrm{PM}_{2.5}, \mathrm{PM}_{10}, \mathrm{SO}_{2}, \mathrm{CO}$ and $\mathrm{NO}_{2}$ had overall higher concentrations in the cold periods and lower concentrations in the warm periods. The peaks of these air pollutants were not evident in the cold period from 2017-2018. The 
164

165

166

167

168

169

170

171

172

173

concentrations of $\mathrm{O}_{3}$ were higher in the warm periods than in the cold periods. The time series of the meteorological parameters of mean temperature, mean relative humidity, mean wind speed and sunshine time are shown in Supplemental Figure 2.

The correlations between the air pollutants and meteorological parameters are shown in Table 2. Strong correlations were detected between any two of the air pollutants except for $\mathrm{O}_{3}$ (all $r>0.600, \mathrm{P}<0.001$ ), while weak correlations were detected between the air pollutants and the meteorological parameters or between any two meteorological parameters except for between the mean temperature and the concentration of $\mathrm{O}_{3}$.

\section{Exposure-response analysis}

A single-pollutant model was applied to investigate the association between a single air pollutant concentration and the number of outpatient visits for ILI when adjusted for the meteorological parameters due to the multicollinearity among the various air pollutants and the sample size during December 2017 to March 2018. Considering the abnormal peak of ILI cases during the cold period from 2017-2018, two models were constructed, one based on the time series from January 2015 to November 2017 (non-outbreak period) and the other based on the time series from December 2017 to March 2018 (outbreak period).

The associations between the outpatient visits for ILI and air pollutants analyzed by the generalized additive Poisson regression model are shown in Figure 3. AQI, $\mathrm{PM}_{2.5}, \mathrm{PM}_{10}, \mathrm{CO}$ and $\mathrm{O}_{3}$ on lag0 days, as well as $\mathrm{SO}_{2}$ and $\mathrm{NO}_{2}$ on lag1 days, were significantly associated with an increased risk of outpatient visits for ILI, which increased by $1.19 \%(\mathrm{RR}=1.012,95 \% \mathrm{CI}: 1.010$ -

1.014), 1.44\% (RR=1.014, 95\% CI: 1.012-1.017), 1.26\% (RR=1.012, 95\% CI: 1.011-1.014), 
185

186

$132.58 \%(\mathrm{RR}=3.765,95 \% \mathrm{CI}: 3.216-4.408), 10.01 \%(\mathrm{RR}=1.105,95 \% \mathrm{CI}: 1.092-1.119)$ and

$4.94 \%(\mathrm{RR}=1.051,95 \% \mathrm{CI}: 1.043-1.059)$, respectively, for a $10 \mu \mathrm{g} / \mathrm{m}^{3}$ increase in the nonoutbreak period. During the outbreak period, the associations changed. For most air pollutants, the association between air pollutant and the number of ILI visits was weakened compared to the non-outbreak period. On lag0 days, the associations between the concentrations of $\mathrm{PM}_{2.5}$ $(\mathrm{RR}=0.971,95 \% \mathrm{CI}: 0.963-0.979), \mathrm{SO} 2(\mathrm{RR}=0.892,95 \% \mathrm{CI}: 0.840-0.948)$ and $\mathrm{CO}(\mathrm{RR}=0.306$, 95\% CI: 0.153-0.612) and the decreased risk of ILI outpatient visits was significant.

Interestingly, on lag2 days, all the pollutants were significantly associated with a reduced risk of outpatient visits for ILI except for $\mathrm{O}_{3}$.

The results of the exposure-response analysis for outpatient visits for ILI associated with continuous air pollutants (lag1 for $\mathrm{AQI}, \mathrm{PM}_{2.5}, \mathrm{PM}_{10}, \mathrm{SO}_{2}, \mathrm{NO}_{2}$, and $\mathrm{CO}$; lag0 for $\mathrm{O}_{3}$ ) as spline functions are presented in Figure 4. We did not observe the linear correlations between the number of outpatient visits for ILI and any of air pollutants, which were instead represented by curvilinear relationship. For $\mathrm{AQI}, \mathrm{PM}_{2.5}, \mathrm{SO}_{2}$, and $\mathrm{CO}$, the risk of outpatient visits for ILI decreased with higher concentrations of air pollutants. The curve for $\mathrm{PM}_{10}$ oscillated at a concentration of $0 \mu \mathrm{g} / \mathrm{m}^{3}$, which means that the number of outpatient visits for ILI was not associated with $\mathrm{PM}_{10}$ concentration. The number of outpatient visits for ILI was associated with an increase of $\mathrm{NO}_{2}$ and $\mathrm{O}_{3}$ concentrations.

The associations between AQI and the number of outpatient visits for ILI in the nonoutbreak period were similar among the three age groups, while for $\mathrm{PM}_{2.5}, \mathrm{PM}_{10}, \mathrm{SO}_{2}, \mathrm{NO}_{2}$ and $\mathrm{CO}$, patients aged 15-24 years old and 25-60 years old had higher RRs than patients aged more 
206

207

208

209

210

211

212

213

214

215

216

217

218

219

220

221

222

than 60 years old (Table 3 and Supplemental Figure 3-Supplemental Figure 8). All the air

pollutants except $\mathrm{O}_{3}$ showed the significant associations in the outbreak period with the decreased risk of outpatient visits of for ILI on lag2 days and higher RRs in patients aged 25-60

years old (except for $\mathrm{SO}_{2}$ ). The $\mathrm{O}_{3}$ concentration was associated with the risk of outpatient visits for ILI both in the non-outbreak period and in the outbreak period (Table 3 and Supplemental

\section{Figure 9).}

\section{Discussion}

Many human diseases, especially in cardiovascular and respiratory systems, are associated with short and long-term exposure to the air pollutants (Rajagopalan et al. 2018; Schraufnagel et al. 2018; Schraufnagel et al. 2019). The correlations between air mass trajectories and pollution conditions in Beijing reinforce the necessity of interregional cooperation and control (Li et al. 2018). Air quality has been improved by strengthening of pollution control in recent years. At

the same time, the numbers of ILI cases and outbreaks increased during the winter of 2017-2018 and surpassed levels from previous years observed from weekly surveillance reports of the Chinese National Influenza Center. Therefore, it is vital to have the opportunity to assess the effects of air pollutants on the number of outpatient visits for ILI. In our study, we found that air pollutants were associated with an increased risk of outpatient visits for ILI during the nonoutbreak period of the influenza virus and reduced the risk of outpatient visits during the outbreak period.

This study provides evidence for the associations between air pollutants and the risk of 
227

228

229

230

231

232

233

234

235

236

237

238

239

240

241

242

243

244

245

246

outpatient visits for ILI. It has been confirmed that exposure to ambient air pollutants is closely associated with localized transmission of influenza (Chen et al. 2017). It is estimated that 50-150 $\mu \mathrm{g} / \mathrm{m}^{3}$ increases in $\mathrm{PM}_{2.5}$ concentration caused a nearly one-fold increase in the risk of lower respiratory tract infection (Cohen et al. 2017). In our study, air pollutants were associated with the risk of outpatient visits for ILI over a period without the influenza pandemic, which was consistent with most studies (Chen et al. 2017; Feng et al. 2016; Liang et al. 2014; Liu et al. 2018; Nhung et al. 2017; Zhang et al. 2015). Feng et al. suggested a strong positive relationship between $\mathrm{PM}_{2.5}$ and ILI risk during the flu season after adjusting for potential variables (Feng et al. 2016). Our findings for an association between ambient $\mathrm{PM}_{2.5}$ concentration and the incidence of influenza in Hefei is consistent with previous studies based on data from other individual cities in China. The study by Liu et al. suggested that $\mathrm{SO}_{2}$ and $\mathrm{NO}_{2}$ were not correlated with ILI (Liu et al. 2018), which was opposite to our results. There is no consensus on the effects of $\mathrm{NO}_{2}$ and $\mathrm{SO}_{2}$ on ILI for many reasons, such as regional differences, population specificities (herd susceptibility and age-sex composition), and a component of the complicated mixture (the proportions of $\mathrm{NO}_{2}$ and $\mathrm{SO}_{2}$ in the air pollutants). These associations need to be analyzed and confirmed in the further studies.

Air pollutants have adverse effects on the outpatient visits for ILI during the non-outbreak periods, but this effect was not observed during the outbreak period in our study. Liang et al. suggested that human influenza cases were correlated with $\mathrm{PM}_{2.5}$ concentrations in Beijing from 2008 and 2011 with a potentially long lag time between the peaks of $\mathrm{PM}_{2.5}$ levels and the peaks of influenza cases in 2009 and 2010 (Liang et al. 2014). In one study from Australia, significant 
248

249

250

251

252

253

254

255

256

257

258

259

260

261

262

263

264

265

266

267

268

associations were reported between exposure to air pollution and the incidence of pediatric influenza at a longer lag time of ten days (Xu et al. 2013). However, the causality between $\mathrm{PM}_{2.5}$ concentrations and influenza cases during the outbreak of influenza in 2009 was questionable due to a lag time of over two or three months, which is difficult to explain biologically. A significantly increased risk for outpatient visits for ILI was not detected during the outbreak of influenza during the winter of 2017-2018 in our study. Rather, air pollutants reduced the risk of outpatient visits for ILI and demonstrated a "protective effect". We think this may be related to the use of disposable face masks and reduced outdoor activities, which further reduces the possibility of pathogen transmission. In other words, the good air quality in the winter of 20172018 may increase possibility of transmitting along with reducing the use of disposable face masks and increasing outdoor activities. Some studies have supported the reduction in influenza incidence through public use of face masks at a population level (Rainwater-Lovett et al. 2014; Uchida et al. 2017).

People with other illnesses or less social support are more vulnerable to air pollution.

Women and people aged more than or equal to 60 years demonstrated a higher risk of respiratory disease after $\mathrm{PM}_{2.5}$ exposure (Xu et al. 2016). PM2.5 was strongly associated with ILI risk across all age groups during the flu season, but the association was most pronounced among adults aged 25-59 years old, followed by young adults aged 15-24 years old and elderly individuals aged more than or equal to 60 years old (Feng et al. 2016). In our study, air pollutants were not more associated with ILI patients aged more than 60 years old compared to other age groups. Previous studies on elderly populations reported associations between $\mathrm{PM}_{2.5}$ concentrations and 
269

270

271

272

273

274

275

276

277

278

279

280

281

282

283

284

285

286

287

288

289

hospitalizations for respiratory diseases, including respiratory tract infections, COPD, and

pneumonia (Dominici et al. 2006). The reason may be that elderly people are more likely to suffer from chronic diseases, such as asthma, COPD, diabetes, and cardiovascular disease, which may determine their susceptibility to the short-term exposure to elevated levels of air pollutants (Simoni et al. 2015). During the outbreak of the influenza virus in our study, young and elderly patients had stronger protective effects compared to the patients aged 25-60 years old. This might be related to the fact that young children and elderly individuals would be better protected and spend less time outdoors, which means less exposure to air pollutants. Then they reduced their exposure to each other, especially with the ILI cases and thereby reduced the risk of ILI. At the same time, individuals aged 25-60 years were more likely to be exposed to a substantial portion of their daily dose of air pollution during commuting activities (de Nazelle et al. 2011).

One study reported that $\mathrm{PM}_{2.5}$ was nonlinearly associated with daily ILI risk based on a generalized additive Poisson model, after adjusting for meteorological parameters and seasonal and yearly trends (Feng et al. 2016), which was consistent with our result. This may be related to preventative measures issued by the government and carried out by individuals themselves.

Some limitations exist in our study. First, we only used the data from one hospital, which may be a poor representation of the population and thus introduce selective bias, although the trends of outpatient visits for ILI in our study were similar to the data in the weekly surveillance reports of the Chinese National Influenza Center, which may affect the observed negative association on some lag days during some special periods. Second, periods of more unusual outbreaks of influenza may be required to further confirm the associations between air pollutants 
290

and outpatient visits for ILI or influenza, especially in situations involving heavy air pollution. Third, the study used an ecological design, which may limit the ability to control for potentially relevant confounders, such as individual-level demographic or socio-economic factors.

\section{Conclusions}

We found that air pollutants increased the risk of outpatient visits for ILI during the nonoutbreak period while revealing a protective effect during an outbreak period. These findings expand current knowledge of ILI outpatient visits correlated to air pollutants during the influenza pandemic, which are of great importance for the development of public health policy about air quality during influenza epidemics and pandemics. It's significant to further evaluate the effectiveness of protective measures during the outbreak period and the associations between air pollutants and outpatients visits for ILI under other situations such as coronavirus disease 2019 in future.

\section{List of abbreviations}

$\mathrm{PM}_{2.5}$ : particulate matter with an aerodynamic diameter less than $2.5 \mu \mathrm{m} ; \mathrm{NO}_{2}$, nitrogen dioxide; $\mathrm{SO}_{2}$, sulfur dioxide; COPD, chronic obstructive pulmonary disease; ILI, influenza like illness;

CI, confidence interval

\section{Availability of data and materials}

The dataset used and analyzed during the current study has been uploaded to PeerJ. 


\section{Acknowledgements}

313 None

\section{References}

Chen G, Zhang W, Li S, Zhang Y, Williams G, Huxley R, Ren H, Cao W, and Guo Y. 2017. The impact of ambient fine particles on influenza transmission and the modification effects of temperature in China: A multi-city study. Environ Int 98:82-88. 10.1016/j.envint.2016.10.004

Chen Y, Zang L, Du W, Xu D, Shen G, Zhang Q, Zou Q, Chen J, Zhao M, and Yao D. 2018. Ambient air pollution of particles and gas pollutants, and the predicted health risks from long-term exposure to PM2.5 in Zhejiang province, China. Environ Sci Pollut Res Int 25:23833-23844. 10.1007/s11356-018-2420-5

Cohen AJ, Brauer M, Burnett R, Anderson HR, Frostad J, Estep K, Balakrishnan K, Brunekreef B, Dandona L, Dandona R, Feigin V, Freedman G, Hubbell B, Jobling A, Kan H, Knibbs L, Liu Y, Martin R, Morawska L, Pope CA, 3rd, Shin H, Straif K, Shaddick G, Thomas M, van Dingenen R, van Donkelaar A, Vos T, Murray CJL, and Forouzanfar MH. 2017. Estimates and 25-year trends of the global burden of disease attributable to ambient air pollution: an analysis of data from the Global Burden of Diseases Study 2015. Lancet 389:1907-1918. 10.1016/S0140-6736(17)30505-6

Croft DP, Zhang W, Lin S, Thurston SW, Hopke PK, Masiol M, Squizzato S, van Wijngaarden E, Utell MJ, and Rich DQ. 2018. The Association between Respiratory Infection and Air Pollution in the Setting of Air Quality Policy and Economic Change. Ann Am Thorac Soc 16:321-330. 10.1513/AnnalsATS.201810$6910 \mathrm{C}$

de Nazelle A, Nieuwenhuijsen MJ, Anto JM, Brauer M, Briggs D, Braun-Fahrlander C, Cavill N, Cooper AR, Desqueyroux H, Fruin S, Hoek G, Panis LI, Janssen N, Jerrett M, Joffe M, Andersen ZJ, van Kempen E, Kingham S, Kubesch N, Leyden KM, Marshall JD, Matamala J, Mellios G, Mendez M, Nassif H, Ogilvie D, Peiro R, Perez K, Rabl A, Ragettli M, Rodriguez D, Rojas D, Ruiz P, Sallis JF, Terwoert J, Toussaint JF, Tuomisto J, Zuurbier M, and Lebret E. 2011. Improving health through policies that promote active travel: a review of evidence to support integrated health impact assessment. Environ Int 37:766-777. 10.1016/j.envint.2011.02.003

Dominici F, Peng RD, Bell ML, Pham L, McDermott A, Zeger SL, and Samet JM. 2006. Fine particulate air pollution and hospital admission for cardiovascular and respiratory diseases. JAMA 295:1127-1134. 10.1001/jama.295.10.1127

Feng C, Li J, Sun W, Zhang Y, and Wang Q. 2016. Impact of ambient fine particulate matter (PM2.5) exposure on the risk of influenza-like-illness: a time-series analysis in Beijing, China. Environ Health 15:17. 10.1186/s12940-016-0115-2

Horne BD, Joy EA, Hofmann MG, Gesteland PH, Cannon JB, Lefler JS, Blagev DP, Korgenski EK, Torosyan N, Hansen GI, Kartchner D, and Pope CA, 3rd. 2018. Short-Term Elevation of Fine Particulate Matter Air 
Pollution and Acute Lower Respiratory Infection. Am J Respir Crit Care Med 198:759-766. 10.1164/rccm.201709-18830C

Huang L, Zhou L, Chen J, Chen K, Liu Y, Chen X, and Tang F. 2016. Acute effects of air pollution on influenzalike illness in Nanjing, China: A population-based study. Chemosphere 147:180-187. 10.1016/j.chemosphere.2015.12.082

Li H, Duan F, Ma Y, He K, Zhu L, Ma T, Ye S, Yang S, Huang T, and Kimoto T. 2018. Case study of spring haze in Beijing: Characteristics, formation processes, secondary transition, and regional transportation. Environ Pollut 242:544-554. 10.1016/j.envpol.2018.07.001

Li R, Jiang N, Liu Q, Huang J, Guo X, Liu F, and Gao Z. 2017. Impact of Air Pollutants on Outpatient Visits for Acute Respiratory Outcomes. Int J Environ Res Public Health 14:pii:E47. 10.3390/ijerph14010047

Liang Y, Fang L, Pan H, Zhang K, Kan H, Brook JR, and Sun Q. 2014. PM2.5 in Beijing - temporal pattern and its association with influenza. Environ Health 13:102. 10.1186/1476-069X-13-102

Liu XX, Li Y, Qin G, Zhu Y, Li X, Zhang J, Zhao K, Hu M, Wang XL, and Zheng X. 2018. Effects of air pollutants on occurrences of influenza-like illness and laboratory-confirmed influenza in Hefei, China. Int $J$ Biometeorol 63:51-60. 10.1007/s00484-018-1633-0

Liu XX, Li Y, Qin G, Zhu Y, Li X, Zhang J, Zhao K, Hu M, Wang XL, and Zheng X. 2019. Effects of air pollutants on occurrences of influenza-like illness and laboratory-confirmed influenza in Hefei, China. Int $J$ Biometeorol 63:51-60. 10.1007/s00484-018-1633-0

Lu B, Wang Y, Zhu Z, Zhang Z, Dong T, Li F, Gao Y, Du X, and Qu Z. 2020. Epidemiological and genetic characteristics of influenza virus and the effects of air pollution on laboratory-confirmed influenza cases in Hulunbuir, China, from 2010 to 2019. Epidemiol Infect 148:e159. 10.1017/S0950268820001387

Nhung NTT, Amini H, Schindler C, Kutlar Joss M, Dien TM, Probst-Hensch N, Perez L, and Kunzli N. 2017. Short-term association between ambient air pollution and pneumonia in children: A systematic review and meta-analysis of time-series and case-crossover studies. Environ Pollut 230:1000-1008. 10.1016/j.envpol.2017.07.063

Rainwater-Lovett K, Chun K, and Lessler J. 2014. Influenza outbreak control practices and the effectiveness of interventions in long-term care facilities: a systematic review. Influenza Other Respir Viruses 8:74-82. 10.1111/irv.12203

Rajagopalan S, Al-Kindi SG, and Brook RD. 2018. Air Pollution and Cardiovascular Disease: JACC State-of-theArt Review. J Am Coll Cardiol 72:2054-2070. 10.1016/j.jacc.2018.07.099

Schraufnagel DE, Balmes JR, Cowl CT, De Matteis S, Jung SH, Mortimer K, Perez-Padilla R, Rice MB, RiojasRodriguez H, Sood A, Thurston GD, To T, Vanker A, and Wuebbles DJ. 2018. Air Pollution and Noncommunicable Diseases: A Review by the Forum of International Respiratory Societies' Environmental Committee, Part 1: The Damaging Effects of Air Pollution. Chest. 10.1016/j.chest.2018.10.042

Schraufnagel DE, Balmes JR, Cowl CT, De Matteis S, Jung SH, Mortimer K, Perez-Padilla R, Rice MB, RiojasRodriguez H, Sood A, Thurston GD, To T, Vanker A, and Wuebbles DJ. 2019. Air Pollution and Noncommunicable Diseases: A Review by the Forum of International Respiratory Societies' Environmental Committee, Part 2: Air Pollution and Organ Systems. Chest 155:417-426. 10.1016/j.chest.2018.10.041

Simoni M, Baldacci S, Maio S, Cerrai S, Sarno G, and Viegi G. 2015. Adverse effects of outdoor pollution in the elderly. J Thorac Dis 7:34-45. 10.3978/j.issn.2072-1439.2014.12.10

Song C, He J, Wu L, Jin T, Chen X, Li R, Ren P, Zhang L, and Mao H. 2017. Health burden attributable to ambient 
PM2.5 in China. Environ Pollut 223:575-586. 10.1016/j.envpol.2017.01.060

Strosnider HM, Chang HH, Darrow LA, Liu Y, Vaidyanathan A, and Strickland MJ. 2018. Age-Specific Associations of Ozone and PM2.5 with Respiratory Emergency Department Visits in the US. Am J Respir Crit Care Med. 10.1164/rccm.201806-11470C

Su W, Wu X, Geng X, Zhao X, Liu Q, and Liu T. 2019. The short-term effects of air pollutants on influenza-like illness in Jinan, China. BMC Public Health 19:1319. 10.1186/s12889-019-7607-2

Uchida M, Kaneko M, Hidaka Y, Yamamoto H, Honda T, Takeuchi S, Saito M, and Kawa S. 2017. Effectiveness of vaccination and wearing masks on seasonal influenza in Matsumoto City, Japan, in the 2014/2015 season: An observational study among all elementary schoolchildren. Prev Med Rep 5:86-91. 10.1016/j.pmedr.2016.12.002

Xia X, Zhang A, Liang S, Qi Q, Jiang L, and Ye Y. 2017. The Association between Air Pollution and Population Health Risk for Respiratory Infection: A Case Study of Shenzhen, China. Int J Environ Res Public Health 14:pii:E950. 10.3390/ijerph14090950

Xu Q, Li X, Wang S, Wang C, Huang F, Gao Q, Wu L, Tao L, Guo J, Wang W, and Guo X. 2016. Fine Particulate Air Pollution and Hospital Emergency Room Visits for Respiratory Disease in Urban Areas in Beijing, China, in 2013. PLoS One 11:e0153099. 10.1371/journal.pone.0153099

Xu Z, Hu W, Williams G, Clements AC, Kan H, and Tong S. 2013. Air pollution, temperature and pediatric influenza in Brisbane, Australia. Environ Int 59:384-388. 10.1016/j.envint.2013.06.022

Zhang A, Zhou F, Jiang L, Qi Q, and Wang J. 2015. Spatiotemporal analysis of ambient air pollution exposure and respiratory infections cases in Beijing. Cent Eur J Public Health 23:73-76. 10.21101/cejph.a4247

Zheng Q, Liu H, Zhang J, and Chen D. 2018. The effect of ambient particle matters on hospital admissions for cardiac arrhythmia: a multi-city case-crossover study in China. Environ Health 17:60. 10.1186/s12940018-0404-z 


\section{Figure Legends}

413 Figure 1. Time series of outpatient visits for influenza like illness (number of daily cases) at

414 fever clinics in Peking University Third Hospital, Beijing during the study period.

Figure 2. Time series of air pollutants (daily mean values) in Beijing during the study period. The air pollutants involved were $\mathrm{AQI}, \mathrm{PM}_{2.5}, \mathrm{PM}_{10}, \mathrm{SO}_{2}, \mathrm{CO}, \mathrm{NO}_{2}$, and $\mathrm{O}_{3}$.

Figure 3. Percent change of outpatient visits for ILI under different day-specific lags for a 10 $\mathrm{mg} / \mathrm{m}^{3}$ increase in air pollutants during the non-outbreak period and during the outbreak period.

A, AQI during the non-outbreak period; $\mathrm{B}$, AQI during the outbreak period; $\mathrm{C}, \mathrm{PM}_{2.5}$ during the non-outbreak period; $\mathrm{D}, \mathrm{PM}_{2.5}$ during the outbreak period; $\mathrm{E}, \mathrm{PM}_{10}$ during the non-outbreak period;

F, $\mathrm{PM}_{10}$ during the outbreak period; $\mathrm{G}, \mathrm{SO}_{2}$ during the non-outbreak period; $\mathrm{H}, \mathrm{SO}_{2}$ during the during the outbreak period. The study course was classified into two periods: a non-outbreak period from January 2015 to November 2017 and an outbreak period from December 2017 to March 2018.

Figure 4. The response-exposure curve of the relationship between air pollutants and outpatient visits for influenza-like illness on the concurrent day by GAM analysis. The solid line indicates 
433 the estimated mean percentage change in daily outpatient visits, and the dotted lines represent 434 twice the standard error. 


\section{Table $\mathbf{1}$ (on next page)}

Demographic characteristics of outpatient visits of influenza like illness at fever clinic in the hospital from January 1, 2015 to March 31, 2018 
1 Table 1. Demographic characteristics of outpatient visits of influenza like illness at fever clinic in 2 the hospital from January 1, 2015 to March 31, 2018

\begin{tabular}{lcccc}
\hline Variable & No. & Median & IQR & Range \\
\hline ILI cases & 35862 & 17 & $12-28$ & $1-295$ \\
15-24 years & 9963 & 5 & $3-8$ & $0-95$ \\
25-60 years & 19324 & 8 & $5-14$ & $1-203$ \\
$>60$ years & 6575 & 4 & $2-6$ & $0-45$ \\
\hline
\end{tabular}

3

4

5 


\section{Table 2 (on next page)}

Spearman correlation coefficients between daily air pollutant concentrations and the meteorological parameters in the study

${ }^{a}$ indicates the $p$ value $>0.05$ 
1 Table 2. Spearman correlation coefficients between daily air pollutant concentrations and the meteorological parameters in the study

\begin{tabular}{|c|c|c|c|c|c|c|c|c|c|c|}
\hline Parameters & AQI & PM2.5 & PM10 & $\mathrm{SO}_{2}$ & $\mathrm{CO}$ & $\mathrm{NO}_{2}$ & $\mathrm{O}_{3}$ & $\begin{array}{c}\text { Mean } \\
\text { temperature }\end{array}$ & $\begin{array}{c}\text { Mean relative } \\
\text { humidity }\end{array}$ & $\begin{array}{c}\text { Mean wind } \\
\text { speed }\end{array}$ \\
\hline PM2.5 & 0.896 & & & & & & & & & \\
\hline PM10 & 0.795 & 0.800 & & & & & & & & \\
\hline $\mathrm{SO}_{2}$ & 0.440 & 0.553 & 0.558 & & & & & & & \\
\hline $\mathrm{CO}$ & 0.709 & 0.855 & 0.630 & 0.627 & & & & & & \\
\hline $\mathrm{NO}_{2}$ & 0.612 & 0.720 & 0.649 & 0.654 & 0.782 & & & & & \\
\hline $\mathrm{O}_{3}$ & 0.181 & $-0.044^{a}$ & $\begin{array}{c}0.041 \\
\mathrm{a}\end{array}$ & $\begin{array}{c}- \\
0.313\end{array}$ & $\begin{array}{c}- \\
0.301\end{array}$ & $\begin{array}{c}- \\
0.366\end{array}$ & & & & \\
\hline Mean temperature & 0.191 & $\begin{array}{c}-0.022 \\
\mathrm{a}\end{array}$ & $\begin{array}{c}0.001 \\
\mathrm{a}\end{array}$ & $\begin{array}{c}- \\
0.519\end{array}$ & $\begin{array}{c}- \\
0.225\end{array}$ & $\begin{array}{c}- \\
0.276\end{array}$ & 0.796 & & & \\
\hline $\begin{array}{c}\text { Mean relative } \\
\text { humidity }\end{array}$ & 0.449 & 0.502 & 0.196 & $\begin{array}{c}- \\
0.169\end{array}$ & 0.501 & 0.262 & $\begin{array}{c}-0.001 \\
\text { a }\end{array}$ & 0.304 & & \\
\hline Mean wind speed & $\begin{array}{c}- \\
0.363\end{array}$ & -0.411 & -0.275 & $\begin{array}{c}- \\
0.145\end{array}$ & $\begin{array}{c}- \\
0.516\end{array}$ & $\begin{array}{c}- \\
0.570\end{array}$ & 0.232 & $0.019^{a}$ & -0.484 & \\
\hline Sunshine time & $\begin{array}{c}- \\
0.191\end{array}$ & -0.392 & -0.145 & $\begin{array}{c}- \\
0.132\end{array}$ & $\begin{array}{c}- \\
0.494\end{array}$ & $\begin{array}{c}- \\
0.286\end{array}$ & 0.400 & 0.207 & -0.553 & 0.303 \\
\hline
\end{tabular}

2 a indicates the $p$ value $>0.05$ 


\section{Table 3 (on next page)}

Relative risk for outpatient visits of influenza like illness by air pollutants in various age groups.

First period, January 2015 to November 2017; second period, from December 2017 to March 2018. 
1 Table 3. Relative risk for outpatient visits of influenza like illness by air pollutants in various age

2 groups.

\begin{tabular}{|c|c|c|c|}
\hline \multirow{2}{*}{ Air pollutants } & \multicolumn{3}{|c|}{ RR $(95 \%$ CI $)$} \\
\hline & $15-24$ years old & $25-60$ years old & $>60$ years old \\
\hline \multicolumn{4}{|l|}{ AQI } \\
\hline First period $(\operatorname{Lag} 0)$ & $1.013(1.009-1.017)$ & $1.014(1.011-1.017)$ & $1.014(1.011-1.017)$ \\
\hline Second period $(\operatorname{Lag} 0)$ & $0.992(0.978-1.006)$ & $0.995(0.986-1.004)$ & $1.005(0.986-1.024)$ \\
\hline Second period (Lag2) & $0.984(0.975-0.994)$ & $0.992(0.986-0.998)$ & $0.988(0.975-1.000)$ \\
\hline \multicolumn{4}{|l|}{$\mathrm{PM}_{2.5}$} \\
\hline First period (Lag0) & $1.015(1.010-1.019)$ & $1.017(1.014-1.020)$ & $1.009(1.003-1.014)$ \\
\hline Second period $(\operatorname{Lag} 0)$ & $0.969(0.953-0.985)$ & $0.969(0.959-0.980)$ & $0.986(0.965-1.009)$ \\
\hline Second period (Lag2) & $0.981(0.971-0.992)$ & $0.988(0.982-0.995)$ & $0.984(0.970-0.998)$ \\
\hline \multicolumn{4}{|l|}{$\mathrm{PM}_{10}$} \\
\hline First period (Lag0) & $1.013(1.011-1.014)$ & $1.014(1.011-1.016)$ & $1.009(1.006-1.013)$ \\
\hline Second period $(\operatorname{Lag} 0)$ & $1.011(1.005-1.018)$ & $1.016(1.008-1.025)$ & $1.023(1.006-1.04)$ \\
\hline Second period (Lag2) & $0.983(0.978-0.987)$ & $0.987(0.98-0.993)$ & $0.983(0.97-0.997)$ \\
\hline \multicolumn{4}{|l|}{$\mathrm{SO}_{2}$} \\
\hline First period (Lag1) & $1.152(1.13-1.174)$ & $1.133(1.115-1.151)$ & $0.920(0.897-0.944)$ \\
\hline Second period (Lag1) & $0.977(0.866-1.102)$ & $1.193(1.111-1.28)$ & $1.028(1.001-1.056)$ \\
\hline Second period (Lag2) & $0.672(0.607-0.743)$ & $0.854(0.806-0.905)$ & $1.044(1.017-1.072)$ \\
\hline \multicolumn{4}{|l|}{$\mathrm{NO}_{2}$} \\
\hline First period (Lag1) & $1.064(1.053-1.076)$ & $1.055(1.046-1.064)$ & $1.033(1.019-1.047)$ \\
\hline Second period (Lag1) & $1.066(1.039-1.094)$ & $1.044(1.027-1.06)$ & $1.024(0.991-1.059)$ \\
\hline Second period (Lag2) & $0.993(0.97-1.016)$ & $0.986(0.972-1.000)$ & $0.977(0.95-1.005)$ \\
\hline \multicolumn{4}{|l|}{$\mathrm{CO}$} \\
\hline First period (Lag0) & $4.556(3.45-6.018)$ & $4.205(3.36-5.263)$ & $3.765(3.216-4.408)$ \\
\hline Second period $(\operatorname{Lag} 0)$ & $1.067(0.28-4.065)$ & $0.222(0.091-0.54)$ & $0.306(0.153-0.612)$ \\
\hline Second period (Lag2) & $0.345(0.148-0.801)$ & $0.382(0.228-0.641)$ & $0.361(0.24-0.543)$ \\
\hline \multicolumn{4}{|l|}{$\mathrm{O}_{3}$} \\
\hline First period $(\operatorname{Lag} 0)$ & $1.002(0.993-1.01)$ & $1.010(1.003-1.016)$ & $1.004(0.995-1.014)$ \\
\hline Second period (Lag0) & $1.009(0.99-1.028)$ & 1.067 (1.054-1.08) & $1.099(1.071-1.128)$ \\
\hline
\end{tabular}

3 First period, January 2015 to November 2017; second period, from December 2017 to March 2018. 
Figure 1

Time series of outpatient visits for influenza like illness (number of daily cases) at fever clinics in Peking University Third Hospital, Beijing during the study period. 


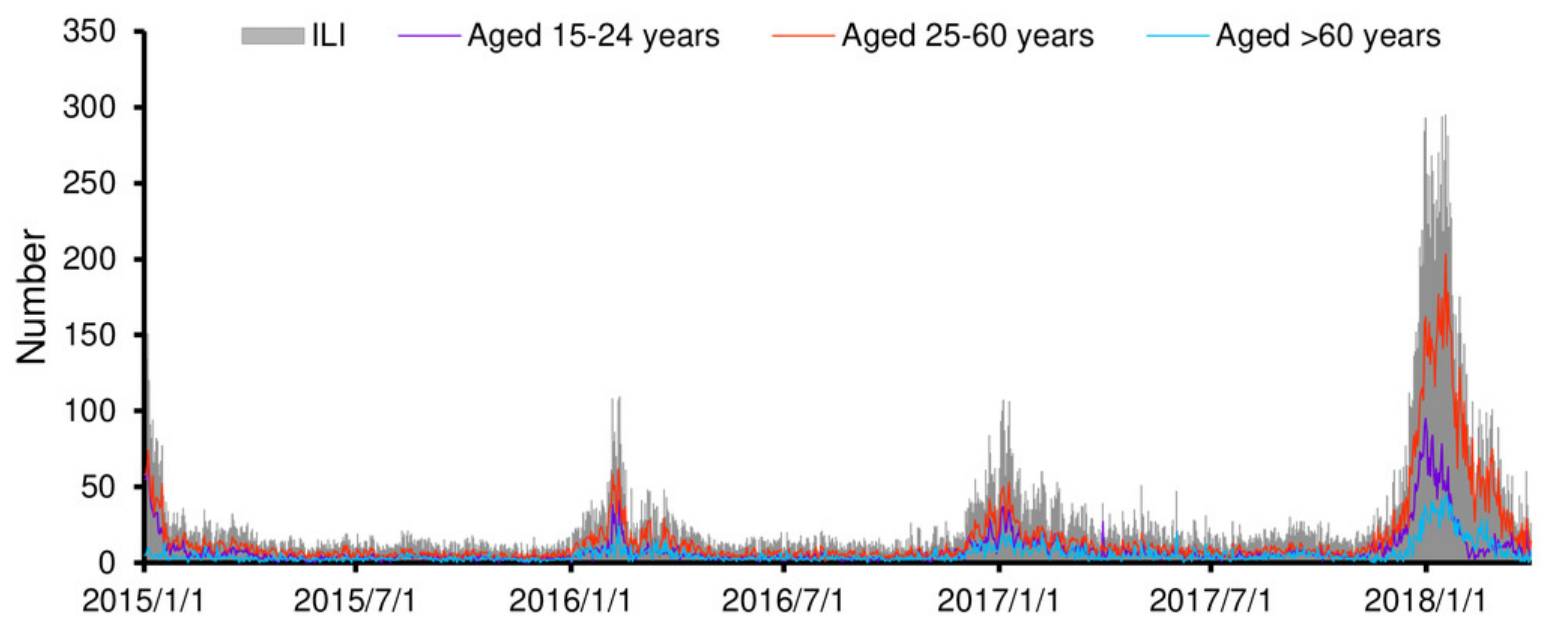


Figure 2

Time series of air pollutants (daily mean values) in Beijing during the study period. The air pollutants involved were $\mathrm{AQI}, \mathrm{PM}_{2.5}, \mathrm{PM}_{10}, \mathrm{SO}_{2}, \mathrm{CO}, \mathrm{NO}_{2}$, and $\mathrm{O}_{3}$. 

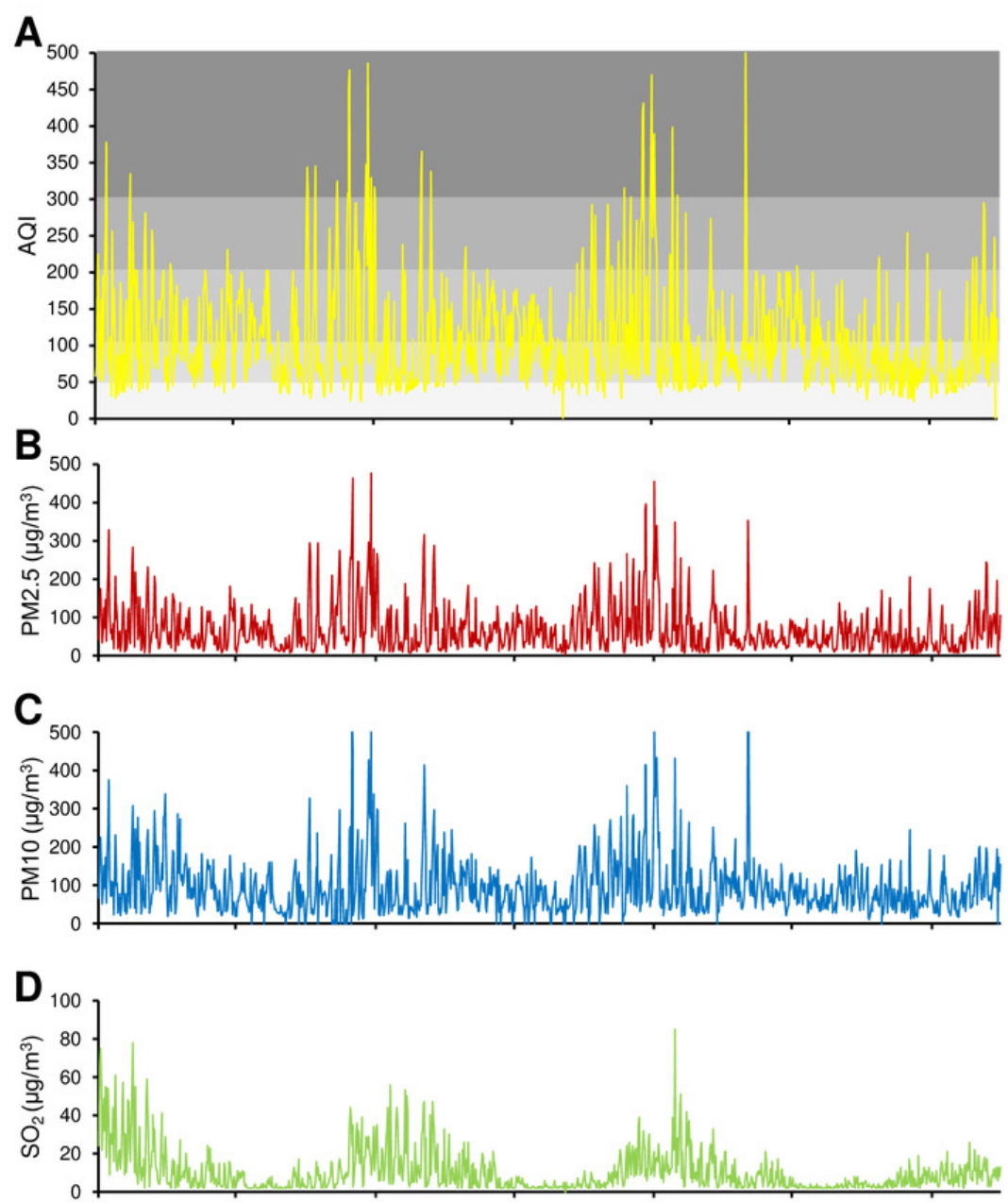

E

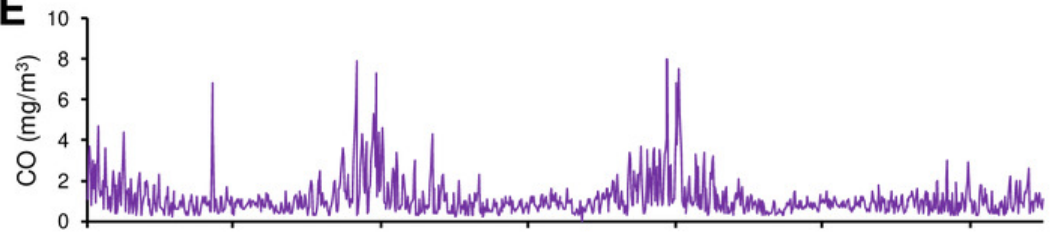

$\mathbf{F}$

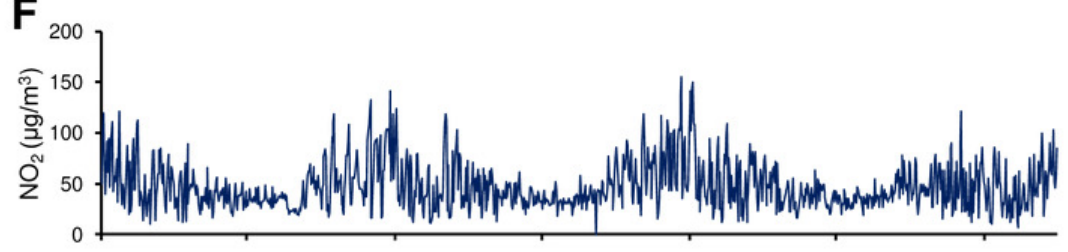

$\mathbf{G}_{350}$

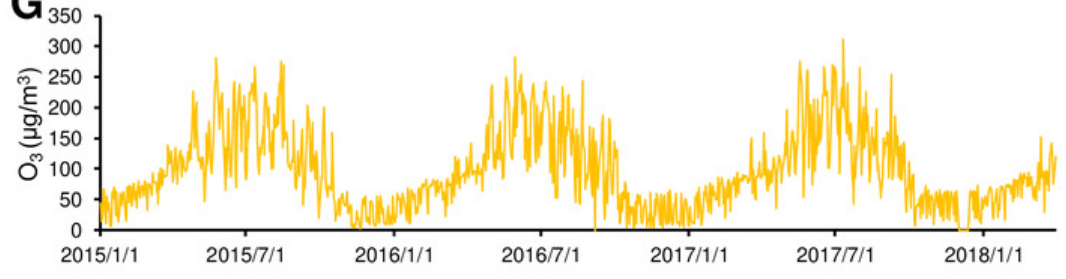




\section{Figure 3}

Percent change of outpatient visits for ILI under different day-specific lags for a 10 $\mathrm{mg} / \mathrm{m}^{3}$ increase in air pollutants during the non-outbreak period and during the outbreak period.

$\mathrm{A}, \mathrm{AQI}$ during the non-outbreak period; $\mathrm{B}, \mathrm{AQI}$ during the outbreak period; $\mathrm{C}, \mathrm{PM}_{2.5}$ during the non-outbreak period; $\mathrm{D}, \mathrm{PM}_{2.5}$ during the outbreak period; $\mathrm{E}, \mathrm{PM}_{10}$ during the non-outbreak period; $\mathrm{F}, \mathrm{PM}_{10}$ during the outbreak period; $\mathrm{G}, \mathrm{SO}_{2}$ during the non-outbreak period; $\mathrm{H}, \mathrm{SO}_{2}$ during the outbreak period; I, CO during the non-outbreak period; J, CO during the outbreak period; $\mathrm{K}, \mathrm{NO}_{2}$ during the non-outbreak period; $\mathrm{L}, \mathrm{NO}_{2}$ during the outbreak period; $\mathrm{G}, \mathrm{SO}_{2}$ during the non-outbreak period; $\mathrm{H}, \mathrm{SO}_{2}$ during the outbreak period; $\mathrm{M}, \mathrm{O}_{3}$ during the nonoutbreak period; $\mathrm{N}, \mathrm{O}_{3}$ during the outbreak period. The study course was classified into two periods: a non-outbreak period from January 2015 to November 2017 and an outbreak period from December 2017 to March 2018. 
A

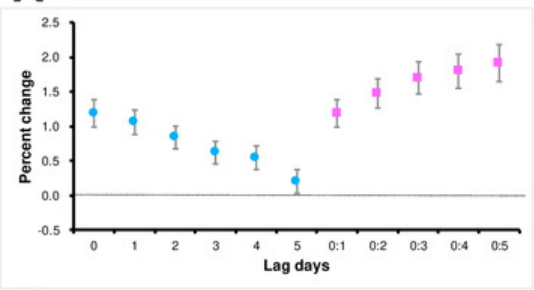

C

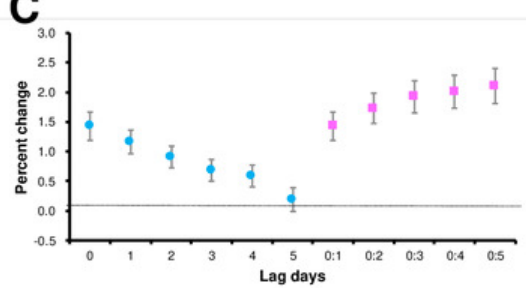

$\mathbf{E}$

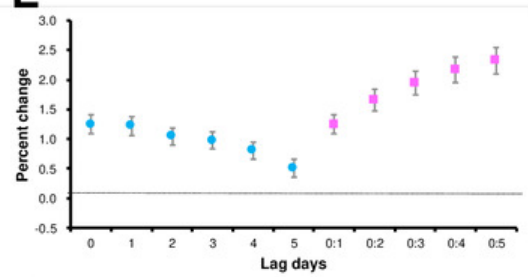

G

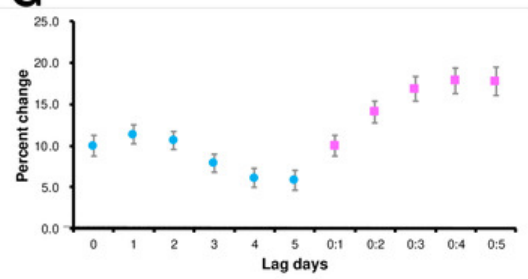

I
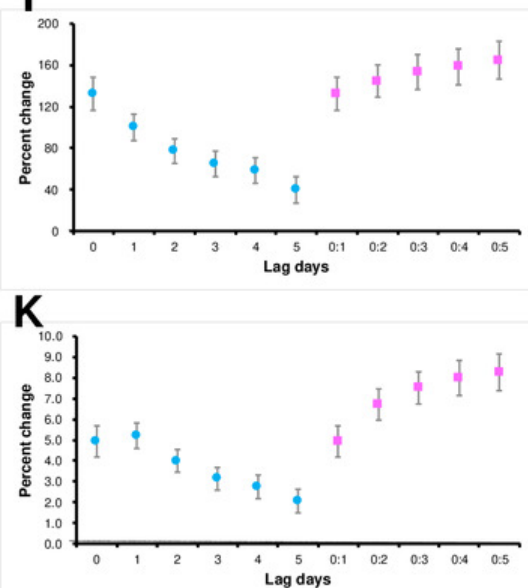

M

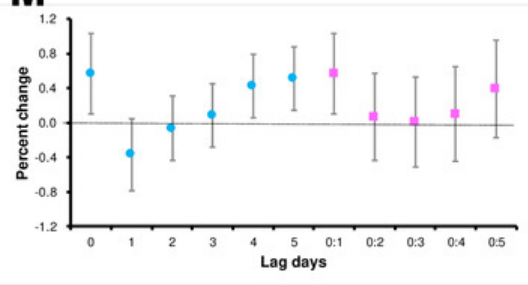

B

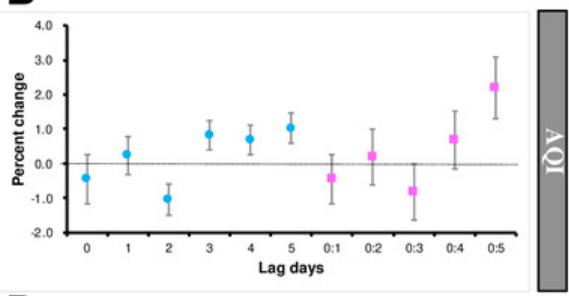

D

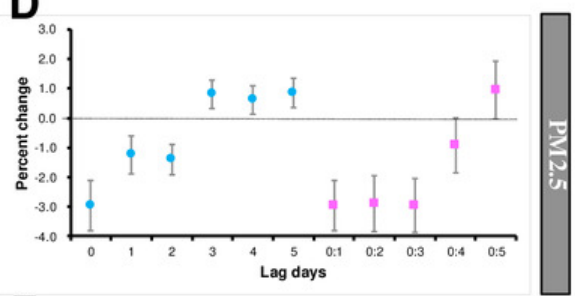

$\mathbf{F}$

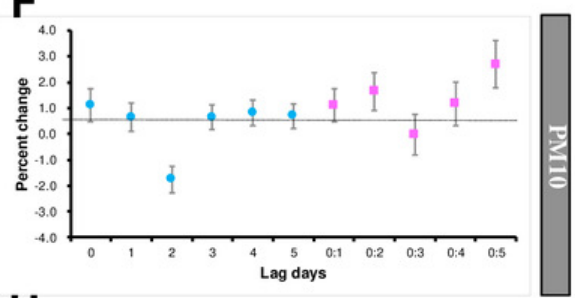

H
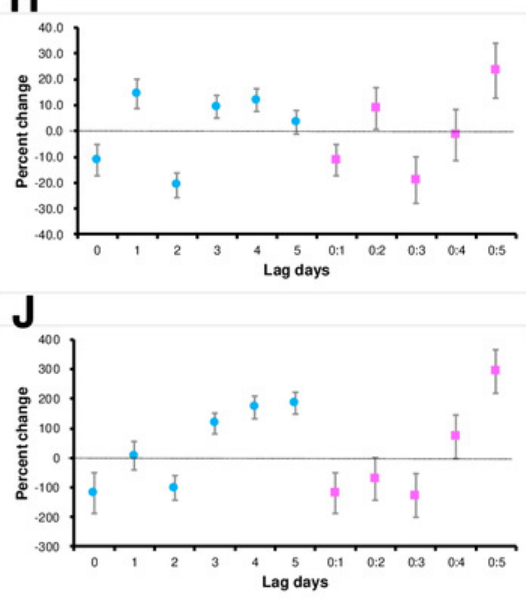

L

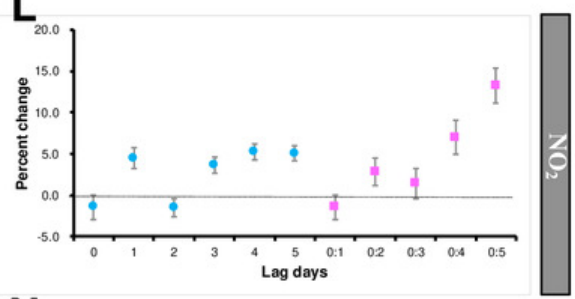

N

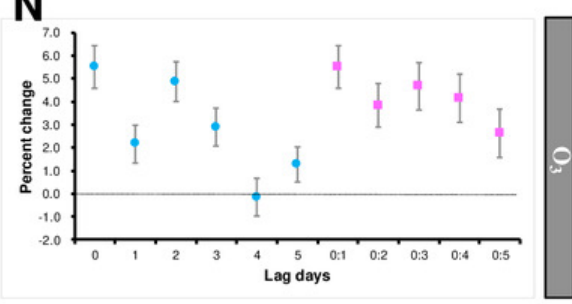


Figure 4

The response-exposure curve of the relationship between air pollutants and outpatient visits for influenza-like illness on the concurrent day by GAM analysis.

The solid line indicates the estimated mean percentage change in daily outpatient visits, and the dotted lines represent twice the standard error. 


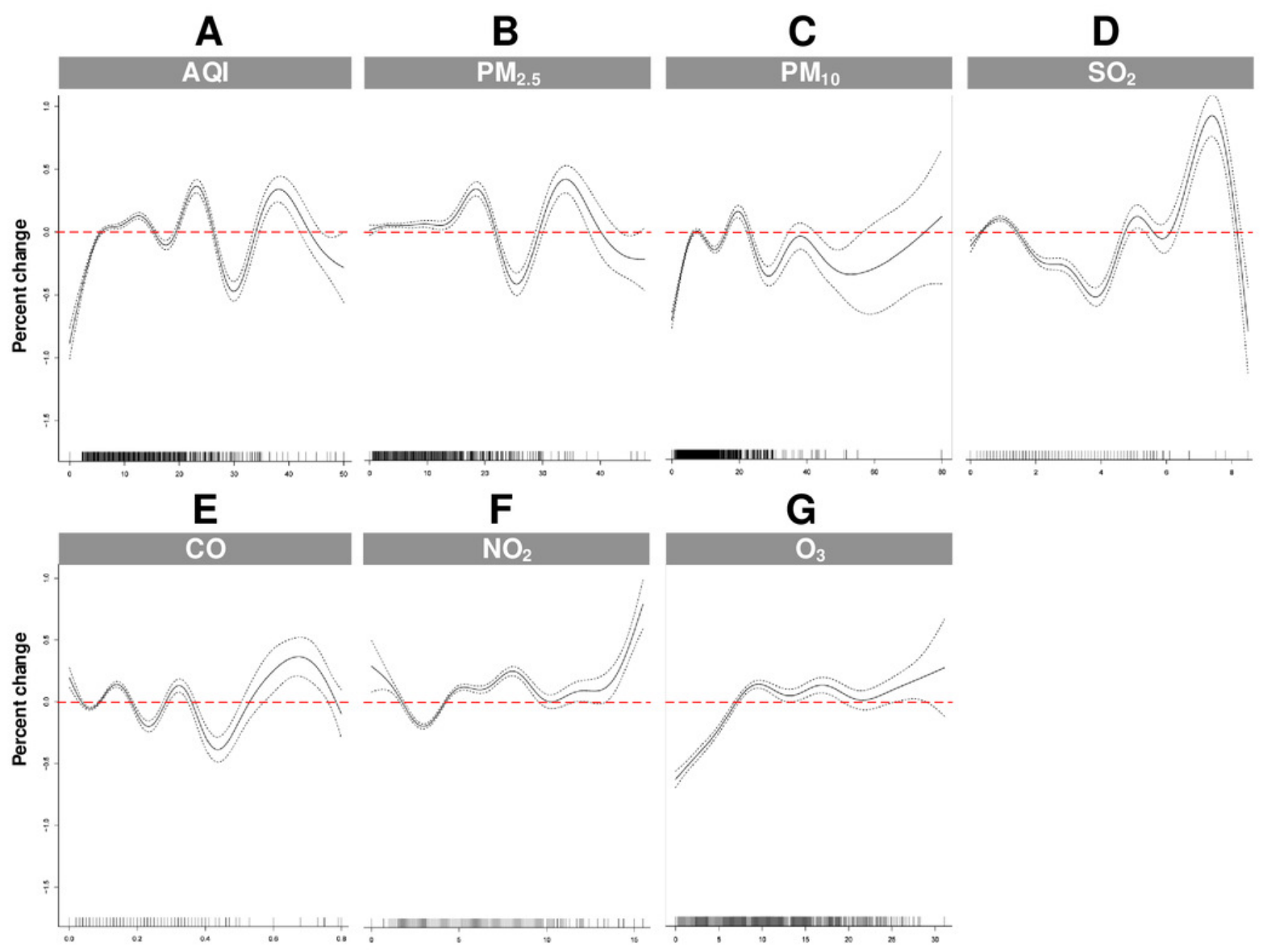

